\title{
Grey Box Non-Linearities Modeling and Characterization of a BandPass BAW Filter
}

\author{
Mohamed MABROUK ${ }^{1,2}$, Mohamed-Ali BOUJEMAA ${ }^{3,4}$, Fethi CHOUBANI $I^{4,5}$ \\ ${ }^{1}$ ISETCOM de Tunis, Cité Technologique des Communications, 2088, Raoued, Ariana, Tunisia \\ ${ }^{2}$ GRESCOM (SUPCOM de Tunis, Université de Carthage, Tunisia) \\ ${ }^{3}$ Faculté des Sciences de Bizerte, Université de Carthage, Tunisia \\ ${ }^{4}$ INNOVCOM (SUPCOM de Tunis, Université de Carthage, Tunisia) \\ ${ }^{5}$ SUPCOM de Tunis, Université de Carthage, Tunisia \\ mohamed.mabrouk@isetcom.rnu.tn
}

Manuscript received December 08, 2015

\begin{abstract}
In this work, the non-linearities of a $3 G / U M T S$ geared BandPass Bulk Acoustic Wave ladder filter composed of five resonators were modeled using non-linear modified Butterworth-Van Dyke model. The non-linear characteristics were measured and simulated, and they were compared and found to be fairly identical. The filter's central frequency is $2.12 \mathrm{GHz}$, the corresponding bandwidth is $61.55 \mathrm{MHz}$, and the quality factor is 34.55 .
\end{abstract}

\section{Keywords}

Band-pass filter, bulk acoustic wave (BAW), modified Butterworth-van Dyke model (MBVD), non-linearities

\section{Introduction}

Bulk Acoustic Wave (BAW) and Thin-Film Bulk Acoustic Resonator (FBAR) based filters have been proven for small footprint high power applications. Owing to their reduced size, and their CMOS process compatibility, they are easily integrable in common RF ICs that operate in the $\mathrm{GHz}$ and sub $\mathrm{GHz}$ spectrum, which is a difficult feat using conventional microstrip technology. Modeling of the nonlinear behavior of BAW filters is an active research area. Several non-linear models are categorized into physical models, such as the non-linear Mason models, and behavioral, like non-linear BVD and MBVD. These article models are usually applied for a single resonator or for a simple structure where no more than two or three resonators are used [1].

For more complex filter structures, and when the individual characteristics of the resonators are unknown and/or influenced by other phenomena such as thermal influence or fabrication defects, the resonator by resonator approach becomes impracticable [2], [3]. A grey box approach is required due to the number of unknowns involved.
In this article, the non-linear modeling of a five resonators BAW filter is described without reference to the parameters of the individual resonator. Only the measured response of the filter is taken into account, in addition to the knowledge of its internal structure. A simple non-linear MBVD model is used to describe the behavior of the individual resonators, and a two-step identification process is used to estimate the small-signal and large-signal model parameters.

\section{BAW Technology}

\subsection{BAW Resonators}

BAW resonators are Micro-Electro-Mechanical Systems (MEMS) devices that perform the function of a classical resonator but without the inconvenience of being bulky. BAW resonators are fully integrable using standard CMOS Very Large Scale Integration (VLSI) process.

A BAW resonator consists of two electrodes, a piezoelectric layer and a Bragg reflector composed from alternating layers of materials with different mechanical properties. BAW technology has several varieties including SMR, FBAR and HBAR. The main difference between those types is the mechanical structure which may, or may not include additional air gaps for example.

In this paper we have studied a Solidly-Mounted Resonator (SMR) based ladder band pass filter.

\subsection{Design and Fabrication}

In SMR resonators, the acoustic reflector consists of alternating layers of two quarter-wave thickness and each low and high impedances respectively as shown in Fig. 1(a) [4]. Usually, these layers of low and high impedance are formed by pairs $\mathrm{SiO}_{2} / \mathrm{AlN}$ or $\mathrm{SiO}_{2} / \mathrm{W}$ [5]. The $\mathrm{SiO}_{2}$ layer has low impedance compared to AlN/W leading to a high coupling factor. The impedance ratio is important 
because it determines the number of pairs needed for a good confinement of the acoustic wave [6]. The higher this ratio, the lower is the number of pairs that will be needed. Therefore, less energy is stored in the reflector resulting in a better coupling factor. Additionally, there will be less loss in the reflector, which allows a better quality factor. For the metals $\mathrm{W}$ and $\mathrm{SiO}_{2}$, the impedance ratio is about 7 . Two pairs of said two layers are sufficient to obtain a reflector with a reflection of $99.98 \%$ in the PCS band. This impedance ratio is lower in the pair $\mathrm{AlN} / \mathrm{SiO}_{2}$ and is of the order of 2.8 which requires more pairs as in the case to realize a reflector $\mathrm{W} / \mathrm{SiO}_{2}$. The layer thicknesses of the reflector also influence the resonance frequency which decreases as the thickness increases. The sensitivity of this frequency with respect to variations in layer thickness is greater as the latter is close to the resonator [7].

In other words, the layers that lie just beneath the resonator have much influence as the last layers have less influence or influence completely negligible. Therefore, they will be taken into account in the design of resonators. Similarly, the number of pairs and the impedance ratio of the reflector materials determine the bandwidth of the reflection coefficient, and so that of the resonator. SMR's are usually modeled using the Butterworth-Van Dyke equivalent lumped model. Larson [8] have introduced (Fig. 2) MBVD model in order to take into account dielectric contact losses. It should be noted that the aforementioned model is a small signal model, which doesn't take into account the soon to be discussed non-linear behavior of the resonator.

The resonator's cross section (Fig. 1b) shows four distinct areas, the substrate, the Bragg reflector, the AIN (Piezoelectric) deposit layer and the electrodes. BAWSMR's are, usually, modeled using the Mason model [9] and the Modified Butterworth-Van Dyke model (MBVD) [8].

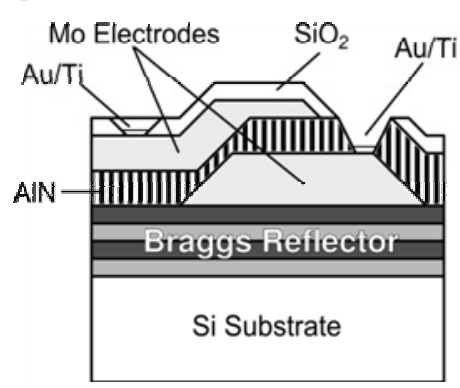

(a)

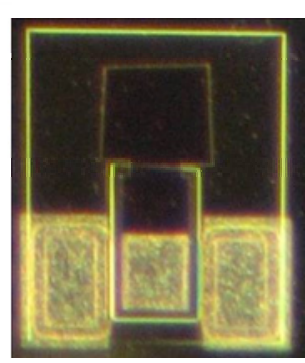

(b)
Fig. 1. Cross section and upper view (layout) of a BAW-SMR resonator.

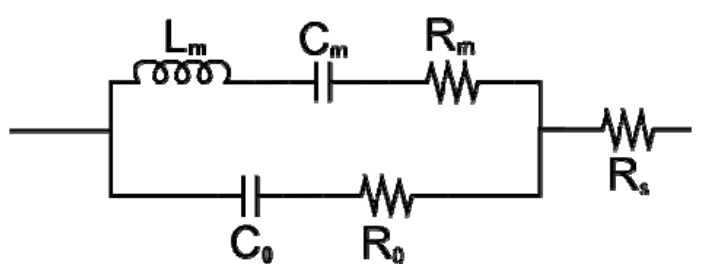

Fig. 2. MBVD model of a BAW resonator.
The complex impedance of a resonator using the MBVD (Fig. 2) model is given below (1).

$$
Z_{\mathrm{e}}=\frac{\left(j L_{\mathrm{m}} \omega-j \frac{1}{C_{\mathrm{m}} \omega}+R_{\mathrm{m}}\right)\left(\frac{1}{j C_{0} \omega}+R_{0}\right)}{\left(j L_{\mathrm{m}} \omega+\frac{1}{j C_{\mathrm{m}} \omega}+\frac{1}{j C_{0} \omega}\right)+R_{\mathrm{m}}+R_{0}}+R_{\mathrm{s}}
$$

where :

$L_{\mathrm{m}}$ and $C_{\mathrm{m}}$ : elements of acoustic resonance frequency,

$R_{\mathrm{m}}$ : mechanical resistor of piezoelectric layer,

$R_{\mathrm{s}}:$ resistor of electrodes,

$C_{0}$ : physical and static capacitor,

$R_{0}:$ dielectric resistor of AlN.

\section{Filter Structure}

The filter is composed of five resonators in a ladder configurations, as illustrated in Fig. 3, with the series $\left(\mathrm{Xs}_{1}\right.$, $\mathrm{Xs}_{2}$ and $\left.\mathrm{Xs}_{3}\right)$ resonators and the parallel resonators $\left(\mathrm{Xp}_{1}\right.$ and $\mathrm{Xp}_{2}$ ) being identical respectively which confers symmetry to the filter's structure. This symmetry is also clear in the filters.

$Z_{0}$ is the reference characteristic impedance (usually $50 \Omega$ ) and $Z_{\mathrm{S}}$ is the impedance of the filter structure:

$$
Z_{\mathrm{s}}=Z_{0}+Z_{1}+Z_{2}
$$

and $Z_{1}$ and $Z_{2}$ are the series and parallel resonator's complex impedance, respectively. The latter are given using the MBVD model (1), with $R_{\mathrm{S}}, R_{0}$ and $C_{0}$ being the same owing to them being mainly dependent on the fabrication process and the used materials only. Thus, the series and parallel resonators can be expressed using the triplets $\left(R_{\mathrm{m} 1}\right.$, $\left.C_{\mathrm{m} 1}, L_{\mathrm{m} 1}\right)$ and $\left(R_{\mathrm{m} 2}, C_{\mathrm{m} 2}, L_{\mathrm{m} 2}\right)$ respectively.

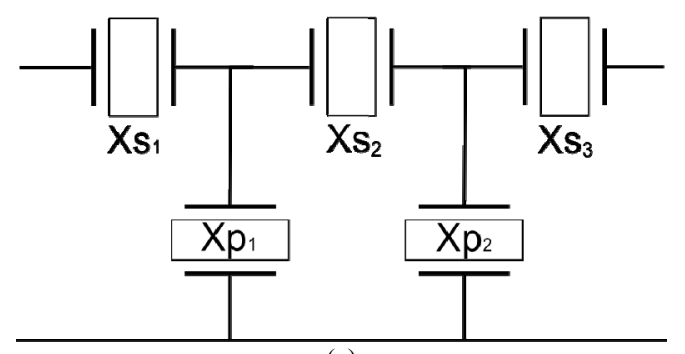

(a)

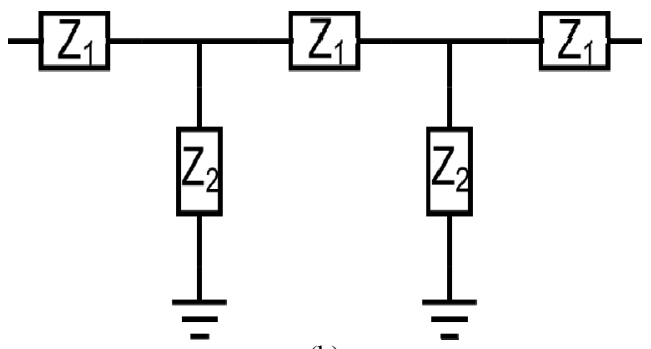

(b)

Fig. 3. Ladder BAW filter architecture (a), Impedance filter structure (b). 
It's possible to obtain the S-matrix using the conversions between $Z$ and $S[10]$ :

$$
\mathbf{S}=\left|\begin{array}{cc}
\frac{Z_{1}^{2}-Z_{2}^{2}-Z_{0}^{2}}{\left(Z_{1}+Z_{0}\right)^{2}-Z_{2}^{2}} & \frac{2 Z_{2}^{2} Z_{0}}{Z_{\mathrm{s}}\left[Z_{1}\left(2 Z_{2}+Z_{\mathrm{s}}\right)+2 Z_{0} Z_{2}\right]} \\
\frac{2 Z_{2}^{2} Z_{0}}{Z_{\mathrm{s}}\left[Z_{1}\left(2 Z_{2}+Z_{\mathrm{s}}\right)+2 Z_{0} Z_{2}\right]} & \frac{Z_{1}^{2}-Z_{2}^{2}-Z_{0}^{2}}{\left(Z_{1}+Z_{0}\right)^{2}-Z_{2}^{2}}
\end{array}\right|
$$

In the present work, we study our fabricated BAW filter (Fig. 3). The aforementioned filter was designed and fabricated in IMEP-LAHC in collaboration with CEA in Grenoble, France. The surface of the fabricated filter is $2 \mathrm{~mm}^{2}$ approximately (Fig. 4), and it operates in the $3 \mathrm{G} / \mathrm{UMTS}$ band (central frequency $2.12 \mathrm{GHz}$, bandwidth 61.55 MHz, quality factor 34.55).

Each resonator is modelized using the MBVD model. Using ADS, we have been able to estimate the model's parameters values using gradient optimization tool. Table 1 contains the values of the series and parallel resonators'

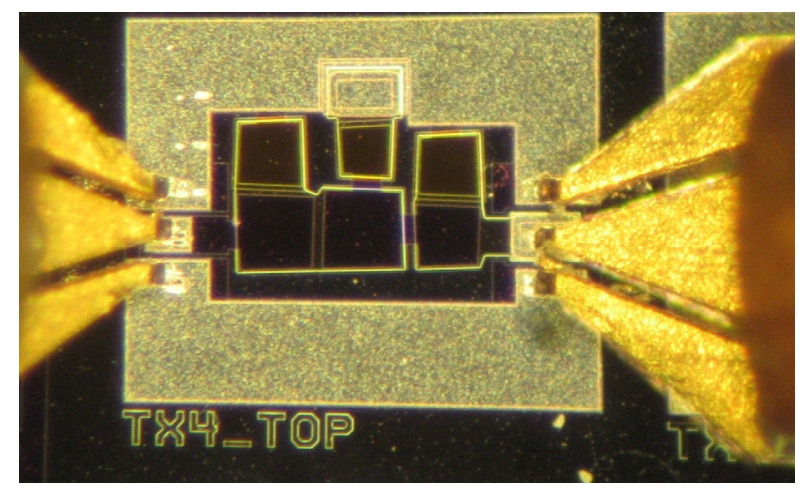

Fig. 4. Photo of the studied ladder BAW filter $\left(2 \mathrm{~mm}^{2}\right)$.

\begin{tabular}{|l|c|c|}
\hline Parameter & $\begin{array}{c}\text { Series } \\
\text { Resonator }\end{array}$ & $\begin{array}{c}\text { Parallel } \\
\text { Resonator }\end{array}$ \\
\hline$R_{\mathrm{m}}[\Omega]$ & 0.9 & 1 \\
\hline$C_{\mathrm{m}}[\mathrm{fF}]$ & 79 & 112 \\
\hline$L_{\mathrm{m}}[\mathrm{nH}]$ & 70 & 53 \\
\hline$R_{0}[\Omega]$ & 4.2 & 3.6 \\
\hline$C_{0}[\mathrm{pF}]$ & 1.6 & 1.69 \\
\hline$R_{\mathrm{s}}[\Omega]$ & 2.6 & 2.6 \\
\hline
\end{tabular}

Tab. 1. Series and parallel resonators' MBVD parameters.

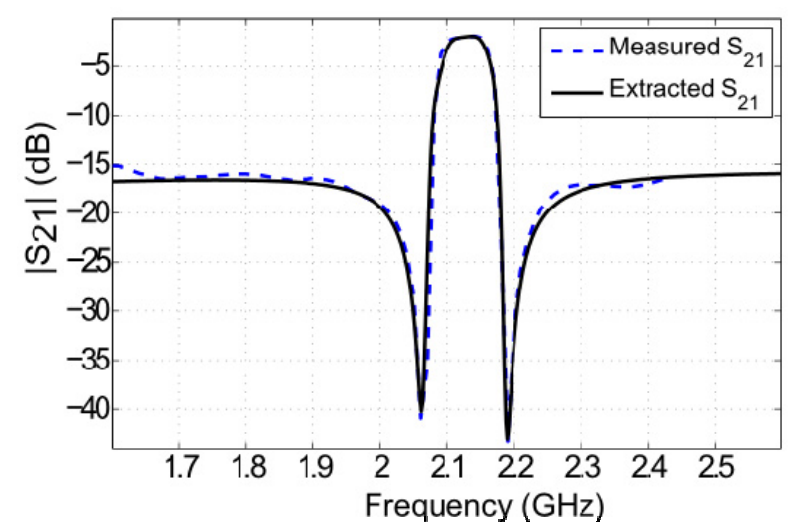

Fig. 5. Measured and extracted small-signal response. parameters. As shown in Fig. 5 the small-signal MBVD parameters were extracted from the measurements. It should be noted that the MBVD model is only accurate for a limited frequency range around the filter's center frequency.

\section{Non-linear Measurements and Characterization}

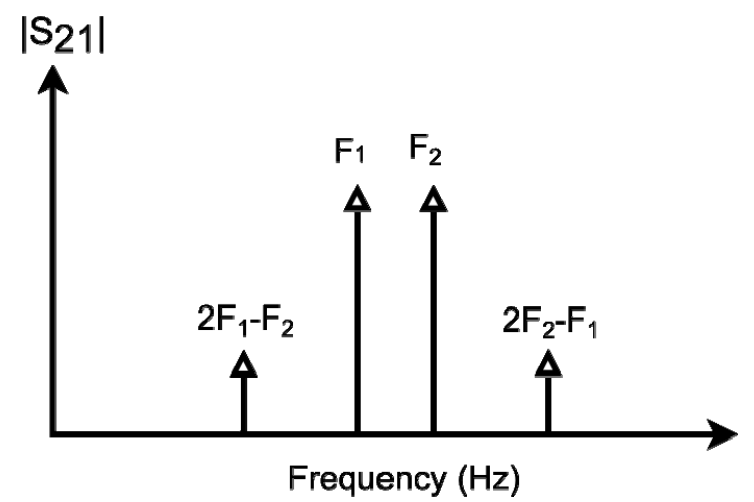

Fig. 6. Frequency spectrum of third order intermodulation products of $F_{1}$ and $F_{2}$ tones injected at the input of the filter.

If excited with high power two tones at frequencies $F_{1}$ and $F_{2}$, a non-linear system exhibits spurious harmonics at its output whose frequencies are located at the mixing product of the two fundamental driving harmonics. The worst spurious harmonics are the Third-Order InterModulation Products ( $3{ }^{\text {rd }}$ IMP) which typically appear at $2 F_{1}-F_{2}$ and $2 F_{2}-F_{1}$ frequencies because they are very close to the fundamentals $F_{1}$ and $F_{2}$ (Fig. 6).

The non-linear measurement chosen for non-linearities modeling is the third order inter-modulation product (IP3). The experimental setup for the measurement is given in Fig. 7.

Two microwave synthesized sources are used for providing two tones at $2.128 \mathrm{GHz}$ and $2.133 \mathrm{GHz}$. Two isolators are used to prevent any inter-modulation phenomena between both sources and before the power combiner. The same high power amplifier is used for bringing high power level at the input of BAW filters. The swept power at the output of both sources is -15 and $-5.0 \mathrm{dBm}$. This means swept input power of amplifier is from -22.0 to $-12.0 \mathrm{dBm}$, and swept power at the input of BAW filter is from $+18.0 \mathrm{dBm}$ to $+28.0 \mathrm{dBm}$. The aggregated measurements are shown in Fig. 8.

Figure 9 is a clearer representation of the evolution of the harmonic power vs the input power.

The measured $3^{\text {rd }}$ point intercept is $38 \mathrm{dBm}$ as shown in Fig. 10.

To take into account the observed non-linearities, the series capacitance $C_{\mathrm{m}}$ and inductance $L_{\mathrm{m}}$ for series and parallel resonators have been considered to be non-linear. 


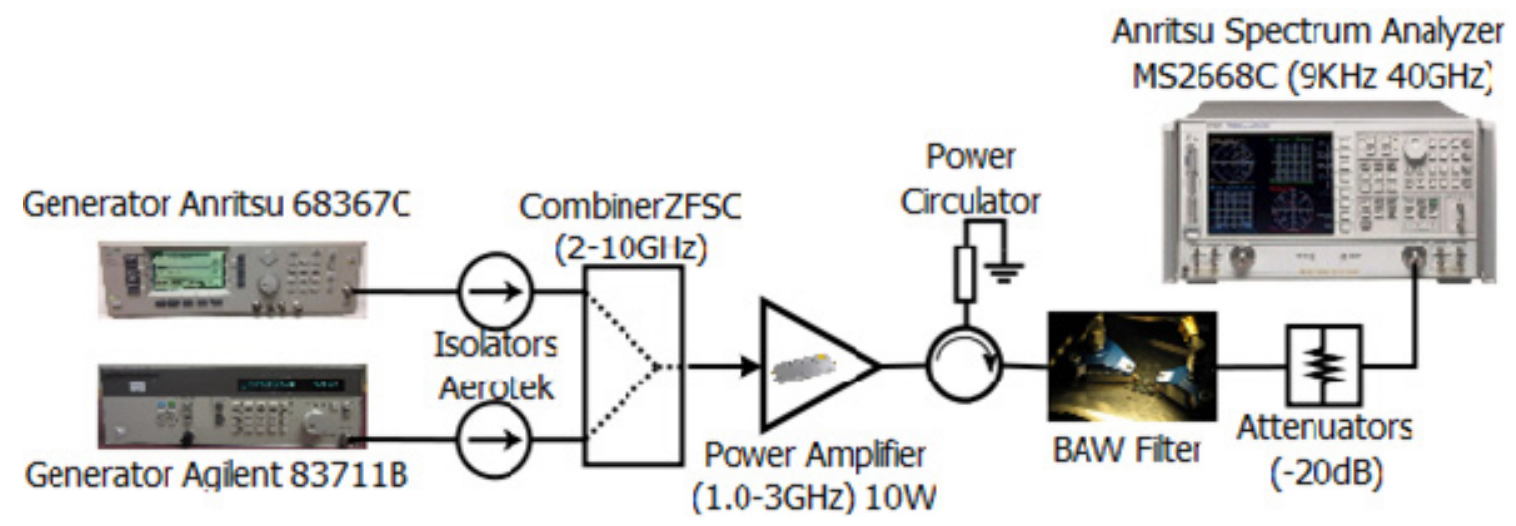

Fig. 7. Non-linearities measurement setup.

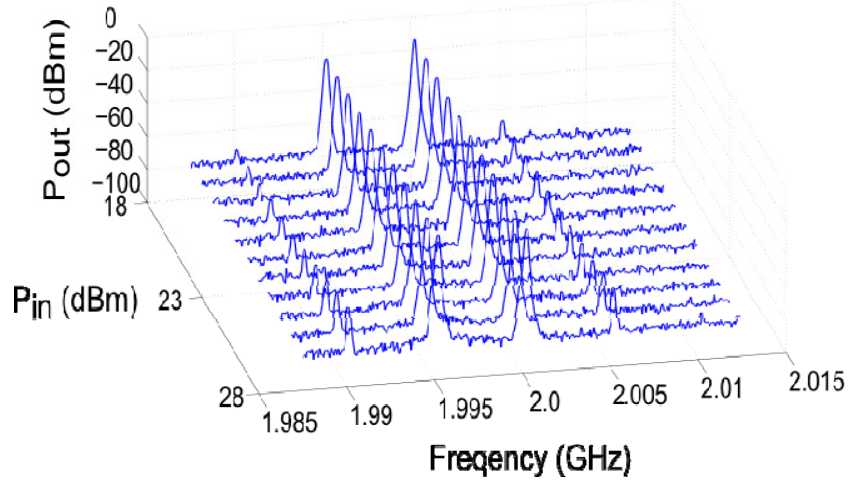

Fig. 8. Power sweeping and measured $3^{\text {rd }}$ IMP results.

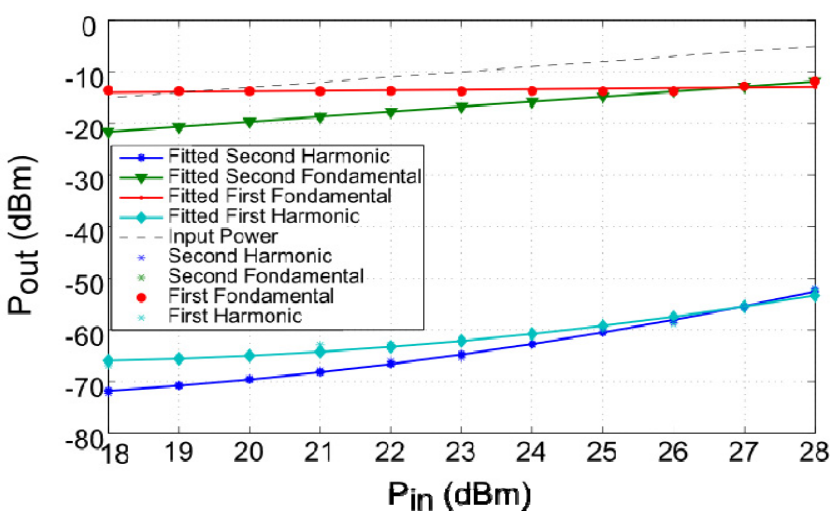

Fig. 9. Measured and fitted Fundamental and harmonics power signals.

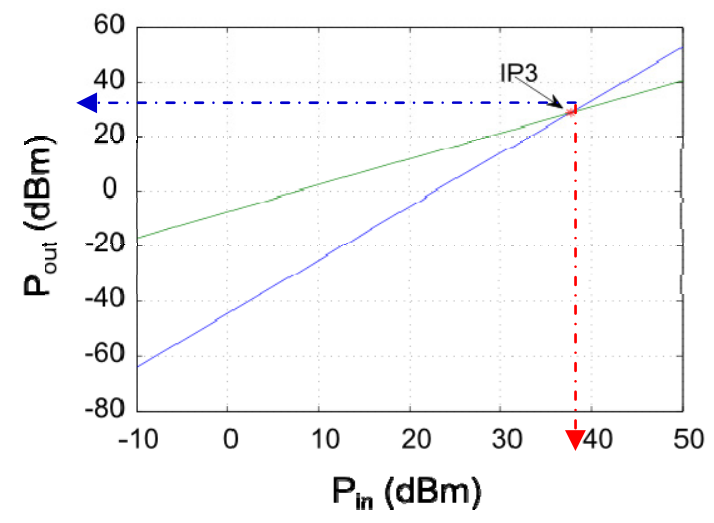

Fig. 10. Intercept Point determination of $3^{\text {rd }}$ IMP.
To characterize the non-linear behavior of the filter, a modified MBVD model where the inductors $L_{\mathrm{m} 1}$ and $L_{\mathrm{m} 2}$ are considered to be non-linear is used. Because almost no physical details about the filter are available (area of the resonators, AlN layer thickness, etc...), an optimization operation is used to estimate the inductors' parameters.

In the relevant literature [2], [3], non-linear components are approximated using current (for inductors and resistors) and voltage (for capacitors) described by polynomial functions, but a characterization object is usually a single resonator. Having a complex filter structure, the only known value is the global input power $P_{\text {in }}$ for each harmonic of the excitation signal. This leads to $P_{\text {in }}$ being present in the inductors' expression.

The chosen inductor non-linear model is given by the following equation:

$$
L_{\mathrm{n} 1}=L\left[\alpha \tanh \left(\beta\left(P_{\mathrm{in}}\right)\right) I+1\right]
$$

where $L_{\mathrm{nl}}$ is the small-signal inductor value, $\alpha$ is a constant that controls the saturation point of the non-linear function, $\beta$ is a function of $P_{\text {in }}$ and $I$ is the RMS value of the current flowing through the inductor. We have obtained the equation (4) using symbolic fitting of the measurement data and the Eureka software package [11]. We have performed some trials starting by polynomial fitting function, after that we have chosen hyperbolic tangent in order to reduce the number of variables to two $\left(\beta_{1}\right.$ and $\left.\beta_{2}\right)$.

\section{Results}

Using ADS, we captured the equivalent circuit for the whole filter (Fig. 11) and used the optimization tool to estimate the coefficients using the measured response.

The simulation results and the measurements are illustrated in Fig. 12. For the measured power range, the output power of the filter correlates closely with the simulated results where the possible error margin should be very small. We have made an OSLT calibration on VNA in order to bring our measurements plans at the input and output of the filter to be characterized. 


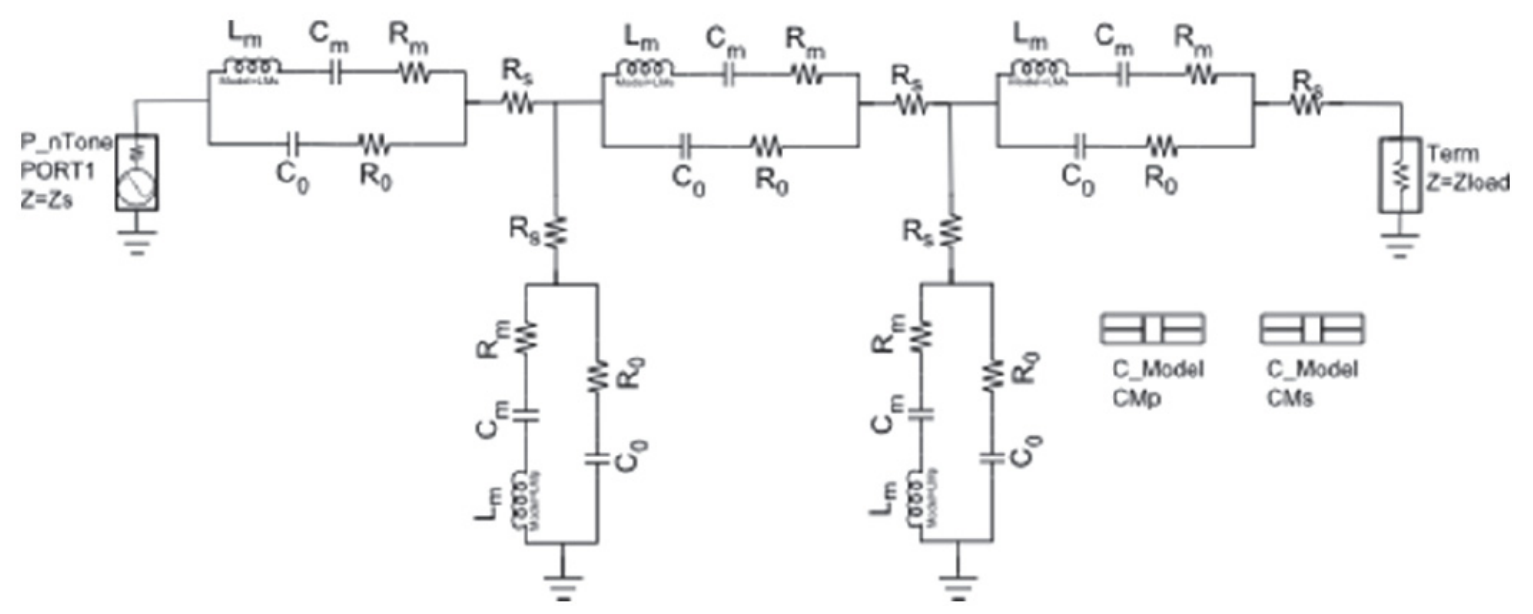

Fig. 11. ADS optimization set-up.

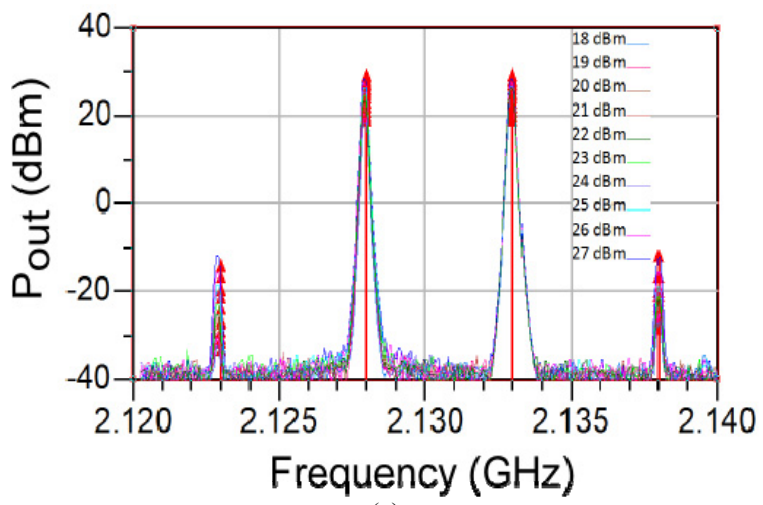

(a)

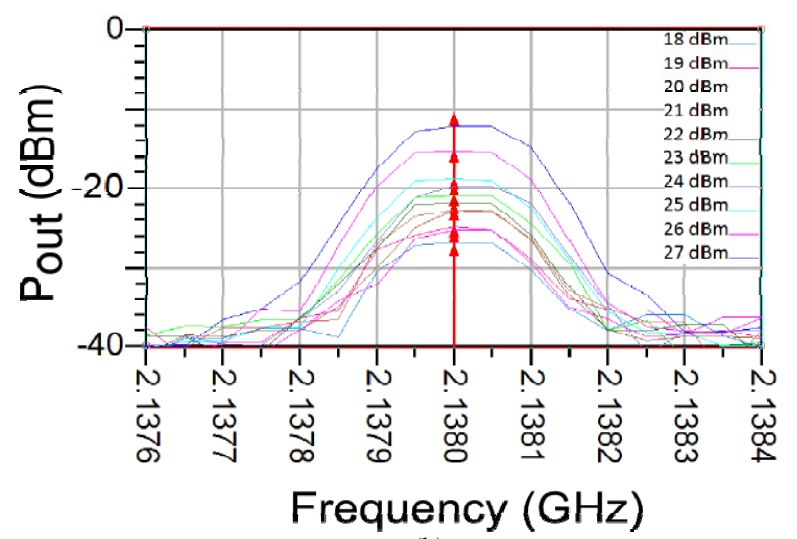

(b)

Fig. 12. (a) Simulated "arrows" versus measured harmonic balance responses for input power ranging from $18.0 \mathrm{dBm}$ to $28.0 \mathrm{dBm}$. (b) Zoomed second harmonic evolution in function of input power.

Table 2 contains the list of the parameter's values for each input power level for both series and parallel resonators. The values of $\alpha_{1}$ and $\alpha_{2}$ were chosen in accordance to the measured magnitude of the spurious harmonics. In the starting trials, we have defined $\alpha_{1}$ and $\alpha_{2}$ as variables, and when we realized that they didn't vary so much we decided to keep $\alpha_{1}$ and $\alpha_{2}$ constant as mentioned in Tab. 2 .

To understand much more the behavior of $\beta_{1}$ and $\beta_{2}$, we have plotted the evolution of $\beta_{1}$ (Fig. 13) as a function

\begin{tabular}{|c|c|c|c|c|}
\hline $\boldsymbol{P}_{\text {in }}[\mathrm{dBm}]$ & $\alpha_{1}$ & $\beta_{1}$ & $\alpha_{2}$ & $\beta_{2}$ \\
\hline 18 & \multirow{11}{*}{30} & -0.095 & \multirow{11}{*}{46} & -0.07 \\
\hline 19 & & -0.081 & & 0.055 \\
\hline 20 & & -0.061 & & -0.05 \\
\hline 21 & & -0.072 & & -0.043 \\
\hline 22 & & -0.092 & & -0.031 \\
\hline 23 & & -0.129 & & -0.011 \\
\hline 24 & & -0.188 & & -0.014 \\
\hline 25 & & -1.053 & & -0.024 \\
\hline 26 & & -1.135 & & -0.031 \\
\hline 27 & & -1.151 & & -0.042 \\
\hline 28 & & -1.134 & & -0.048 \\
\hline
\end{tabular}

Tab. 2. Series and parallel resonators non-linear parameters.

of $P_{\text {in }}$ which behaves like Heaviside function. This behavior could be linked to the transition from a weak non-linear behavior to a stronger non-linearity regime between $24.0 \mathrm{dBm}$ and $25.0 \mathrm{dBm}$ input power.

Also we have plotted $\beta_{2}$ (Fig. 14) that exhibits small variation for the whole power range with some values nearing zero, which indicates a weak non-linear behavior for the parallel resonators. The disparity between the series and parallel resonators non-linear behavior is due to the excitation frequencies choice. Being located within the passing region of the filter, the excitation harmonics power should traverse the series resonators mostly, and thus affecting them more.

Figures 15 and 16 are a graphic representation of the evolution of the values of $L_{\mathrm{m}}$ versus the input power for series and parallel resonator. We note that they are highly dependent on the input power. The parallel resonators seem to have more erratic behavior compared to the series resonators. This can be explained by the reliance of computing the value of $C_{\mathrm{m}}$ as a direct function of $L_{\mathrm{m}}$ which fixes the series resonance frequency.

The obtained results prove that our grey-box approach undertaken in this letter is viable, and would result in a workable model. In addition, the non-linearity parameters were reduced to only four parameters, which reduce the search space for the optimization procedure. 


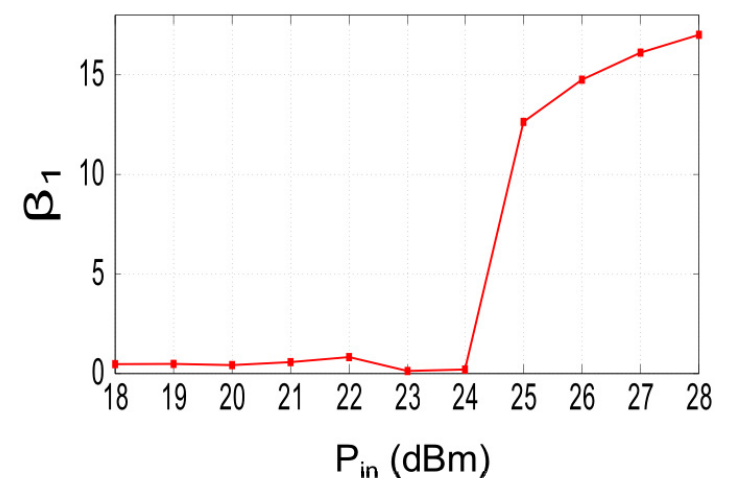

Fig. 13. Evolution of $\beta_{1}$ parameter.

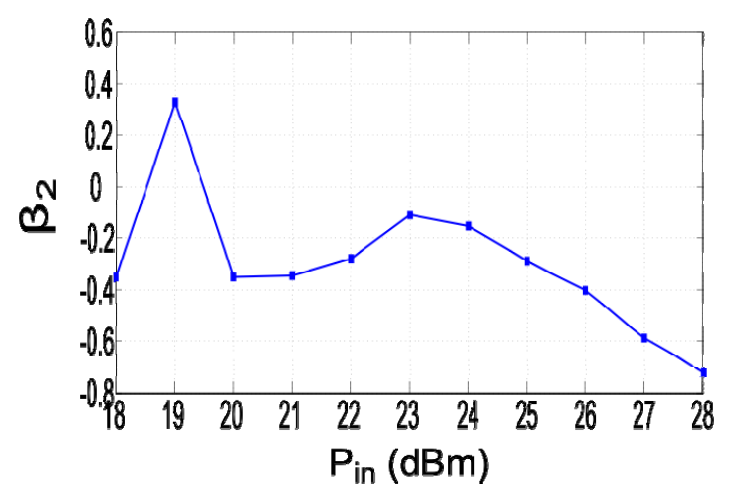

Fig. 14. Evolution of $\beta_{2}$ parameter.

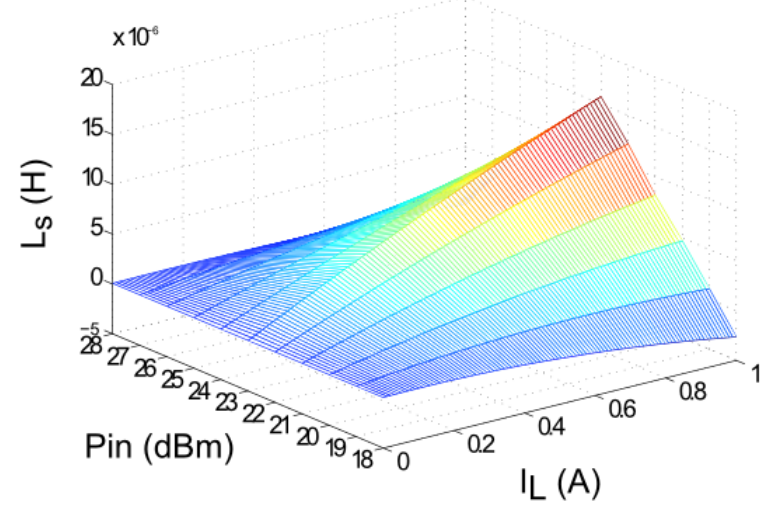

Fig. 15. Series resonator's inductance vs. input power and instant current.

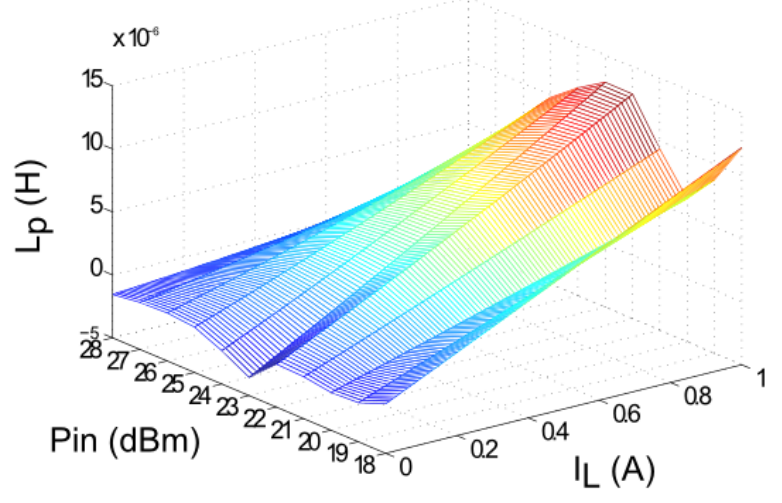

Fig. 16. Parallel resonator's inductance vs. input power and instant current.

\section{Conclusion}

We have carried out a non-linearities modeling of a five resonators ladder BAW filter after using power measurements of transmission response. The obtained fitted model agrees well with the measurements. The use of a non-polynomial non-linear function for describing the non-linearities induced by high power excitation describes better the observed non-linear behavior than the usual polynomial fitting. In addition, the corresponding number of the non-linear parameters was reduced to four for the whole filter which includes two distinct resonator types. The proposed non-linear resonator model is applicable to different structures, with varying number of resonators.

\section{References}

[1] AIGNER, R. SAW and BAW technologies for RF filter applications: A review of the relative strengths and weaknesses. In IEEE International Ultrasonic Symposium (IUS). Beijing (China), 2-5 November 2008, p. 582-589. DOI:10.1109/ULTSYM.2008.0140

[2] COnstantinescu, F., GHEOrGHe, A., Stroe, G., et al. A nonlinear circuit model for a three BAW resonator filter working at high power level. In Proceedings of the 2012 International Conference on Synthesis, Modeling, Analysis and Simulation Methods and Applications to Circuit Design (SMACD). Seville, (Spain), 19-21 September 2012, p. 233-236. DOI: 10.1109/SMACD.2012.6339382

[3] Stroe, G., COnstantinescu, F., GHEORGHE, A., et al. Measurement and modeling of nonlinear effects for power BAW filters with AlN. In $8^{\text {th }}$ International Advanced Topics in Electrical Engineering (ATEE). Bucharest (Romania), 23-25 May 2013, p. 1-4. DOI: 10.1109/ATEE.2013.6563473

[4] MISU, K., IBATA, K., WADAKA, S., et al. Acoustic field analysis of surface acoustic wave dispersive delay lines using inclined chirp IDT. IEICE Trans. Fundam. Electron. Commun. Comput. Sci., $1^{\text {st }}$ May 2007, vol. E90-A, no 5, p. 1014-1020. ISSN: 0916-8508 DOI: 10.1093/ietfec/e90-a.5.1014

[5] MORGAN, D. P. History of SAW devices. In Proceedings of the 1998 IEEE International Frequency Control Symposium. Pasadena (CA, USA), 27-29 May 1998, p. 439-460. DOI: 10.1109/FREQ.1998.717937

[6] Olivares, J., WEGMANN, E., CAPILla, J., et al. Sputtered $\mathrm{SiO}_{2}$ as low acoustic impedance material for Bragg mirror fabrication in BAW resonators. IEEE Transactions on Ultrasonics, Ferroelectrics, and Frequency Control (UFFC), January 2010, vol. 57, no. 1, p. 23-29. ISSN: 0885-3010. DOI: 10.1109/TUFFC.2010.1374

[7] MOREIRA, C. P., SHIRAKAWA, A. A., KERHERVE, E., et al. Design of a fully-integrated BiCMOS/FBAR reconfigurable RF receiver front-end. In Proceedings of the $18^{\text {th }}$ Symposium on Integrated Circuits and Systems Design. Florianopolis (Santa Catarina, Brazil), 4-7 September 2005, p. 138-143. DOI: 10.1109/SBCCI.2005.4286846

[8] LARSON, J. D., RUBY, J. D., BRADlEY, R. C., et al. Power handling and temperature coefficient studies in fbar duplexers for the $1900 \mathrm{MHz}$ PCS band. In IEEE International Ultrasonic Symposium (IUS). San Juan, 22-25 October 2000, vol. 1, p. 869-874. DOI:10.1109/ULTSYM.2000.922680

[9] FELD, D. A. One-parameter nonlinear Mason model for predicting $2^{\text {nd }}$ and $3^{\text {rd }}$ order nonlinearities in BAW devices. In IEEE 
International Ultrasonic Symposium (IUS). Roma (Italy), 20-24 April 2009, p. 1082-1087. DOI:10.1109/ULTSYM.2009.5441599

[10] FRICKEY, D. Conversions between S, Z, Y, H, ABCD, and T parameters which are valid for complex source and load impedances. IEEE Transactions on Microwave Theory and Techniques (MTT), February 1994, vol. 42, no. 2, p. 205-211, ISSN: 0018-9480. DOI: 10.1109/22.275248

[11] DUBCAKOVA, R. Eureqa: software review. Genetic Programming and Evolvable Machines, 2011, vol. 12, no. 2, p. 173-178. DOI: 10.1007/s10710-010-9124-z

\section{About the Authors ...}

Mohamed MABROUK (IEEE Member \# 00370239) was born in 1961 in Aouadna, Menzel-Chaker, Sfax, Tunisia. He received the DEA and PhD Thesis degrees in OOM (Optique Optoélectronique et Microondes) from Institut National Polytechnique of Grenoble (INPG), France in 1988 and 1991, respectively. His doctoral research concerned RF and Microwave Test and Measurements using TRL calibration for MMIC characterization between 2 and $18 \mathrm{GHz}$. From 1995 to 1998, he worked for Teradyne, Inc. (Paris-office and Boston-Factory, MA, USA) as RF/Microwave Applications Engineer, where he developed several ATE turn-key RF test solutions and dedicated trainings for European Semiconductors manufacturers. Since 1999, he is an Assistant Professor at ISETCOM of Tunis, Tunisia. He is currently teaching RF and microwave devices, transceiver equipment and RF/microwave measurements and he was the head of telecommunications department (40 permanent higher education teachers and 15 engineers) till July 2014. He is the head of Tunable RF Devices and Integration Team, GRESCOM Research Laboratory (SUPCOM de Tunis). His current research interests include RFID applications, RF and UWB filters, phase shifters, integrated printed antennas characterization and $\mathrm{RF} /$ microwave non-linearities measurements. Mohamed Mabrouk authored book chapter "RF and Microwave Test of MMICs: from Qualification to Mass Production", in "Advances in Monolithic Microwave Integrated Circuits for Wireless Systems: Modeling and Design Technologies", IGI Global Publishing, Hershey, PA (USA), September 2011. He is author and co-author of over 70 papers in journals and conferences. M. Mabrouk received "Habil- itation Universitaire, HU" (Tunisian National Authorization Degree for Research Supervising) in Telecommunications from the Ecole Polytechnique de Tunisie (EPT). M. Mabrouk is the supervisor of Master of Science degrees (four discussed) and six $\mathrm{PhD}$ thesis (three discussed and three ongoing).

Mohamed-Ali BOUJEMA was born in Bizerte, Tunisia, in 1983. He received the BSc degree in Electronics from Bizerte's Faculty of Sciences in 2007, the MSc degree in Instrumentation and Measurement from the National Institute of Applied Sciences and Technology of Tunis (INSAT) in 2010, the MSc degree in Engineering and Embedded Systems Instrumentation and Measurement from Mater's High Institute of Applied Sciences and Technology (ISSAT) in 2011, Tunisia. He is currently pursuing the Ph.D. degree in the domain of modeling of piezoelectric devices and BAW filters with the Laboratory INNOVCOM (SUPCOM of Tunis), Tunisia.

Fethi CHOUBANI was born in 1961 in Mahdia, Tunisia. He received the electrical engineering diploma from Ecole Nationale d'Ingénieurs de Tunis (ENIT), Tunisia in 1987 and the M. Eng and Ph.D. degrees from Institut National Polytechnique de Toulouse (ENSEEIHT), Toulouse, France in 1988 and 1993, respectively. Since 1993, he has been with SUPCOM, Ecole Supérieure des Communications de Tunis as an Assistant, Associate and then Professor where he taught radiofrequency components and devices, propagation and antennas, and electromagnetic compatibility. His main interests are focused on oscillators and their applications to electromagnetic sensors, EMC, nonlinear devices, modeling of passive, active components and RF techniques and measurements. He has been offered a position of visiting Research Professor in the Department of Electrical and Computer Engineering at the University of Illinois at Urbana-Champaign in 1999 during 3 months, and in Laplace laboratory in ENSEEIHT for one month. He was head of the Telecommunications Department, ESPTT (Tunisia) from 1995 to 1996, director of strategic studies, Tunisia Telecom (Tunisian operator in Telecommunications during 1999-2001, Director of High Institute of Technological studies during 2010-2011 and advisor of ICT Minister during 2012-2014. He has published more than 100 journal and international conference papers. 